\title{
Drag reduction due to recirculating bubble control using plasma actuator on a squareback model
}

\author{
Budiarso $^{1}$, Harinaldi ${ }^{1, *}$, Riza Farrash Karim ${ }^{2}$, and James Julian ${ }^{2}$ \\ ${ }^{1}$ Departement of Mechanical Engineering, Faculty of Engineering Universitas Indonesia. Kampus Baru UI Depok 16424, Indonesia \\ ${ }^{2}$ Fluid Mechanics Laboratory, Faculty of Engineering Universitas Indonesia. Kampus Baru UI Depok 16424, Indonesia
}

\begin{abstract}
Flow control on a squareback object which resembles many engineering related objects is believed to be highly beneficial. One of the flow characteristics behind the object, recirculating bubble, is known to play significant role in pressure distribution. Meanwhile, plasma actuator implementation on such object is still underdeveloped in application basis. This paper focuses on acquiring a deeper understanding of plasma actuator effect on flow phenomenon behind a squareback object, especially on its application to recirculating bubble control in order to reduce drag. The experiment was divided into drag measurement experiment and visualization experiment. The drag measurement result shows that plasma actuator succeeded on reducing drag up to $15.36 \%$ in the lowest Reynolds number. Meanwhile, the visualization experiment shows that plasma actuator has shifted the recirculating bubble position to be closer to the object's wall.
\end{abstract}

\section{Introduction}

The squareback object has a lot shape similarity on its application in the real world such as structures like towers and bridges supports or vehicles like trains and truck trailers. This proves how important the knowledge of the flow at the squareback object really is and thus will provide great benefits. The squareback objects, resembles blunt objects which known to have form drag. This form drag is caused by the flow which seperated in the corner of the object squareback and formed the wake and ultimately give the effect of a large pressure difference on the object [1]. The effects later felt by the objects, such as excessive use of the fuel and the greater stress on structural objects. This condition is what makes an understanding in flow control on squareback object which focuses on its application in drag reduction becomes highly beneficial.

This later confirmed with a limited amount of flow control research on this particular objects. Even though several researches has shown using varied orifice area which correlates to the length of separation zone [2], using pulsed jets [3], and even suction only method [4], the amount of researches which connect the relation between flow control on this object to drag reduction is still difficult to find.

In order to understand what are the factors which contributes to drag force on squareback object, an understanding in flow phenomenon in squareback object is recommended. With the approach of the flow passes through the Backward Facing Step, it was noted there are three areas that affect the flow [5]. The three areas are the zone of reattachment, recirculation, and shear layer. It was also noted that in the area of shear layer, there are several factors which affected it, i.e. mechanism of vortex pairing and rolling, mechanism of vortex shedding, and the coherent structure. This coherent structure which is known to have long lifetime [6] and was made from roll up phase of the unstable separated layer [7], is also known to have correlation in reattachment length reduction. It is believed that coherent structure caused the increasing fluid entrainment in recirculating bubble [8]. This confirmed the importance of recirculating bubble region area to flow control in such object.

Flow control method to which the flow is being controlled is also important. One of the methods for active flow control is Dielectric Barrier Discharge or what is best known as plasma actuator. Plasma actuator in the last decade has gained interest of researchers in flow control field for its advantages in compare with other active flow control methods. Plasma actuator is known for its advantages in having fast responds, no moving components, and low energy consumption [9]. The application of plasma actuator itself on squareback object is not a new thing. Control on the vortex shedding has been done using plasma actuator which resulted in transverse actuator configuration as the best one [10] while another research suggested the placement to be at the upstream of separation point [11].

However, the research on application of plasma actuator as an active flow control on bluff body is still underdeveloped, especially on its application in reducing drag. This is predicted due to the focus of the research is still focusing in the mechanism and the phenomenon of the plasma actuator itself. Therefore, the research development on plasma actuator application is still quite wide and promising.

\footnotetext{
Corresponding author: harinald@eng.ui.ac.id
} 


\section{Methodology}

The main method which was used in this research is experimental method. The experiment itself was divided to two experiments: visualization experiment and drag measurement experiment. Visualization experiment was focused on observing the flow behind squareback object in order to get the recirculating bubble area data. Meanwhile, the drag measurement experiment was objected to acquire the plasma actuator effect on drag reduction as the integrated result of the actuation process. The model which was being used in the experiment was $0.366 \mathrm{~m}$ long, $0.078 \mathrm{~m}$ high, and 0.073 wide. The plasma actuators were placed on the back of the object, given the fact that the freestream came from the front of the object. The electrodes were placed on the top and the bottom of the back side of the object.

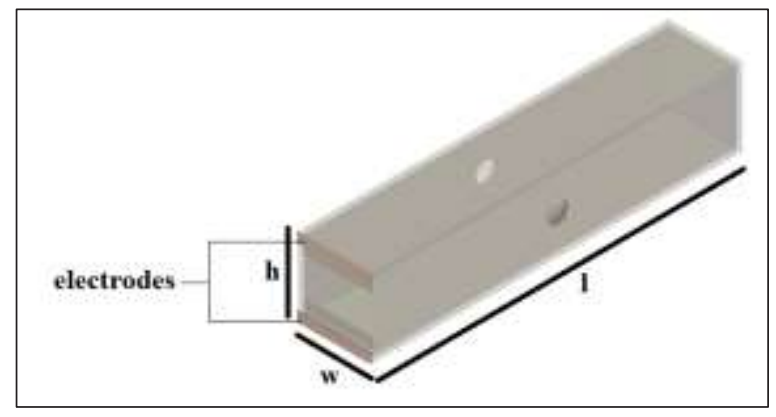

Fig 1. Experiment model

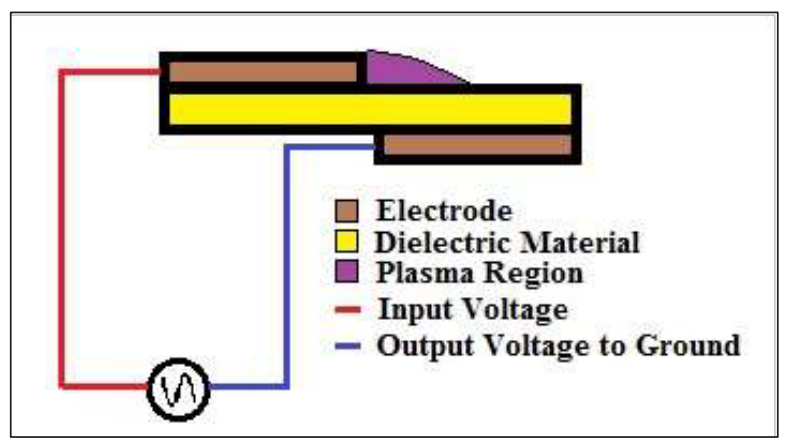

Fig 2. Plasma actuator configuration

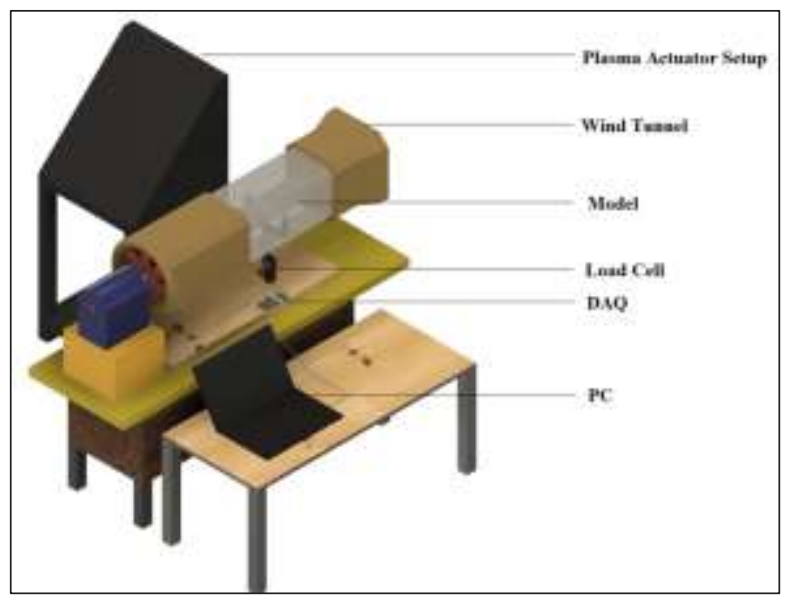

Fig 3. Drag measurement experiment setup

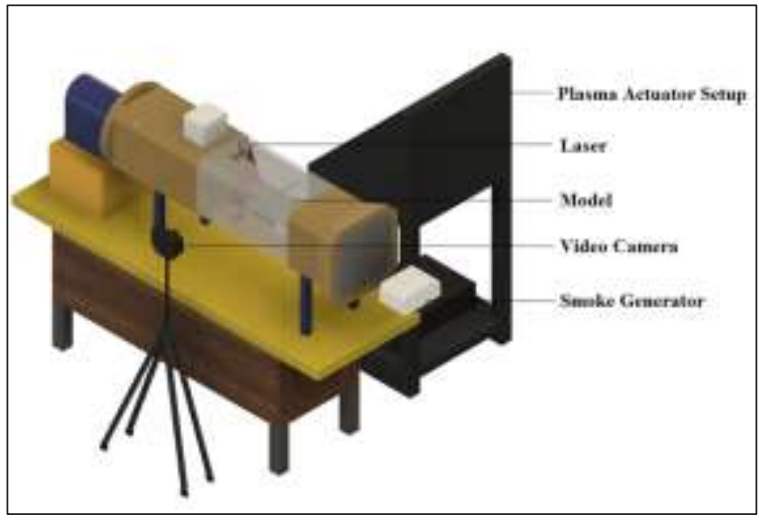

Fig 4. Visualization experiment setup

The plasma configuration is shown on fig. 1. The configuration consists of two electrodes and one dielectric material which made of copper and acrylic consecutively. These electrodes have a thickness of 10 $\mathrm{mm}$ and a width of $50 \mu \mathrm{m}$, while the dielectric has a thickness of $3 \mathrm{~mm}$. To generate plasma with the configuration, it takes a high voltage transformer with the type of step up that is able to generate the voltage of $8 \mathrm{kVp}-\mathrm{p}$ with a current of $0.03 \mathrm{~A}$. Plasma generation is done at a frequency of $10 \mathrm{kHz}$ triangle wave type, with the voltage and the current which kept constant so that it was obtained the power of $120 \mathrm{~W}$.

The drag measurement process was conducted using load cell which connected to DAQ then to the computer to be read. The velocity of the freestream ranged from ReD $=1.3 \times 10^{4}-3.0 \times 10^{4}(2.5 \mathrm{~m} / \mathrm{s}-5.6 \mathrm{~m} / \mathrm{s})$ with the model height being the $\mathrm{D}$. Meanwhile, the visualization technique which was used in this research is smoke visualization. The object was placed within the wind tunnel, and later then being exposed with laser sheet. The smoke was generated with smoke generator which then supplemented to the inlet of the wind tunnel. Video camera later was used to capture the smoke particles which passed through the object and then scattered by the laser sheet. The video later processed frame by frame to acquire the measurement of flow properties which required to calculate the recirculating bubble area.

\section{Results and Discussion}

The first result which was shown in the fig. 5 shows the measurement of drag force which was acquired by the load cell. Due to higher velocity, it is shown that the drag force which was affecting the object to be higher than the lower velocity. The figure shows drag force reduction which was presented due to plasma actuator. On the first velocity variation $(2.5 \mathrm{~m} / \mathrm{s})$ it was clear that the reduction was quite noticeable unlike the second velocity variation $(5.6 \mathrm{~m} / \mathrm{s})$. This drag measurement indeed consisted of form drag and friction drag but due to the plasma configuration which was placed perpendicular to the freestream, it is safe to assume that the drag force reduction result happened due to plasma actuator effect on the form drag alone. It is also noticeable that due to plasma actuator, the acquired data appeared to fluctuate. 
This result can be caused by the plasma actuator perturbation on the vortex shedding which affects the vibration on the load cell.

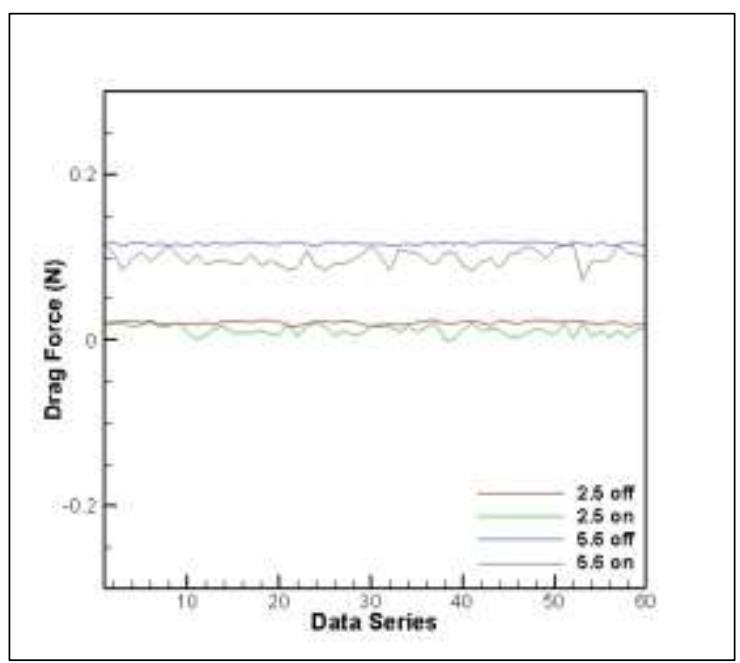

Fig 5. Drag force measurement result

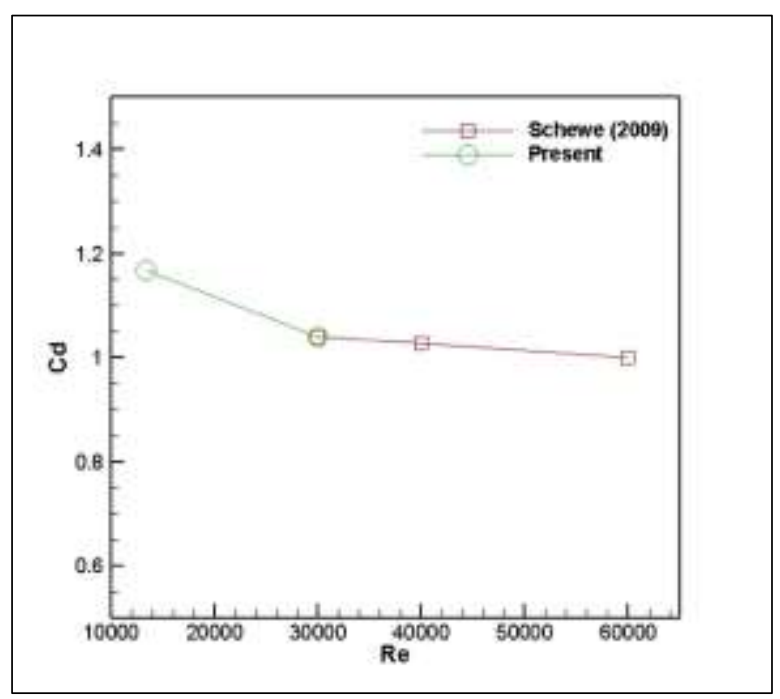

Fig 6. Drag coefficient on varied Reynolds number

Meanwhile, fig. 6 shows the drag coefficient which is the processed data version of drag force measurement. In order to assure that the acquired data was true, the figure also shows the literature data [12]. It is noticeable that the literature data value isn't too farfetched with the acquired data. It also noticeable the descending trend of drag coefficient which appeared both on acquired data and the literature. This can be caused by the domination of the shape of the object to the flow itself which caused by the quadratic ascending of velocity aspect in drag coefficient formula.

The drag reduction percentage graph which was shown on fig. 7 shows the descending trend of drag reduction as the Reynolds number increases. This phenomenon can be caused by the principal work of the plasma actuator itself which depends on the ionizing process of the surrounding gas. As this process needs time, the higher the Reynolds number means the faster the flow velocity, which means the shorter the time that plasma actuator uses to ionize the surrounding gas and later resulted in the descending trend of drag reduction percentage. The analogy of this phenomenon can be illustrated by the bypass factor phenomenon in heat transfer on different tube configurations. The best drag reduction percentage was achieved at the lowest Reynolds number with $15.36 \%$. This trend was also achieved by another experiment [13].

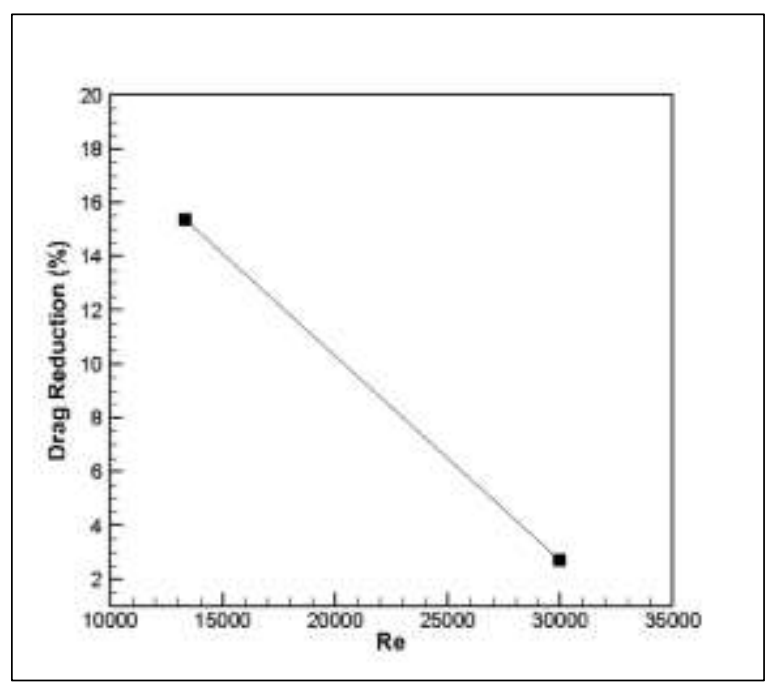

Fig 7. Drag reduction percentage
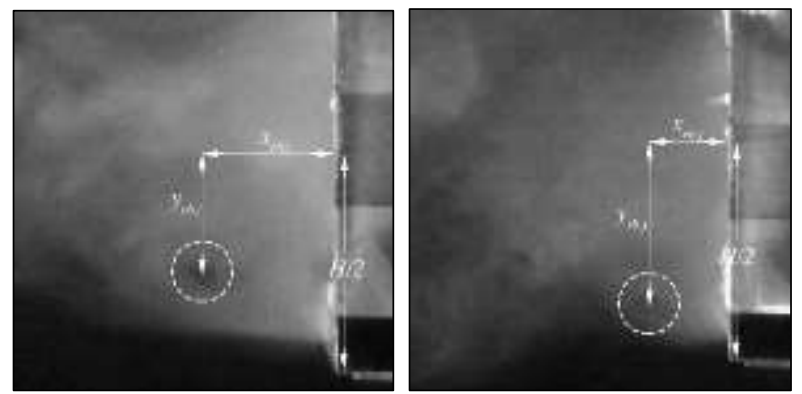

Fig 8. Recirculating bubble assessment method for visualization experiment

Visualization method was initiated by determining the flow assessment method. Fig. 8 shows the assessment method which recognizes the flow. Recirculating bubble is characterized by the center of the circulation which shown by the black hollow structure in the picture. This structure later processed frame by frame to show the mean location of the recirculating bubble both before and after plasma actuator contribution.

The result on fig. 9 shows that the recirculating bubble position shifted due to plasma actuator effect. $X=0$ point was located on the surface of the object's wall. This means that the recirculating bubble has shifted towards the objects wall. This can be caused by the plasma actuator effect on separated flow which passed the edge of the squareback object. This separated flow later perturbed by plasma actuator, then affected the coherent structure. Coherent structure, which was the product of the separated shear layer, is characterized by the large momentum on itself. By the perturbation of the plasma actuator, this property later became even larger, so that the 
coherent structure became even more influential than before. This later influenced the surrounding flow by its ability to entrain. The surrounding flow later influenced by the vorticity of the coherent structure which then formed into recirculating bubble. Due to plasma actuator, this location of recirculating bubble forming later shifted to the object's wall. Later, the area of the recirculating bubble itself shrunk and thus increased the pressure distribution behind the wall. This phenomenon later suggested to be the cause of the drag reduction effect by plasma actuator on the squareback objects

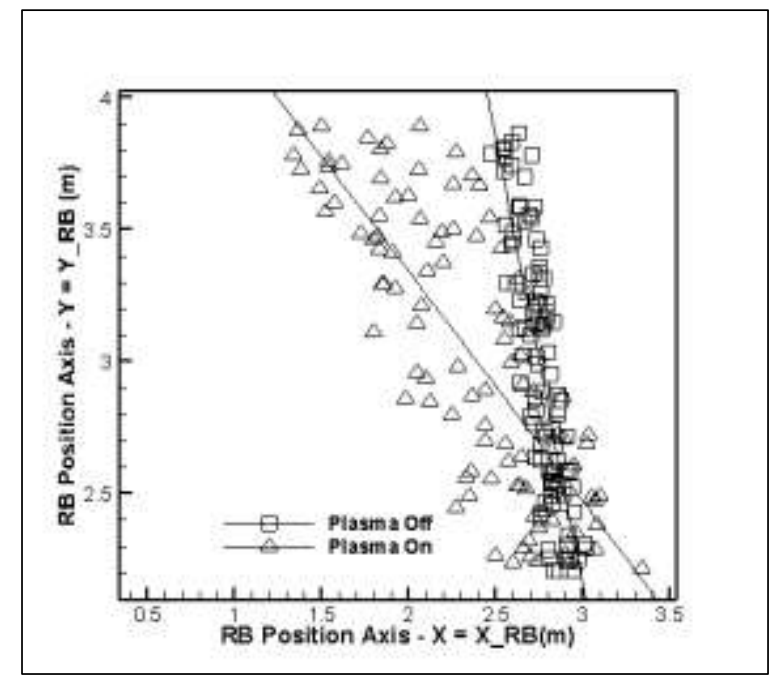

Fig 9. Recirculating bubble position due to plasma actuator

\section{Conclusion}

Plasma actuator has proven to be able to reduce drag on squareback object up to $15.36 \%$ on low Reynolds number. This result is correlated by the visualization result which shows that recirculating bubble position has shifted due to plasma actuator. Recirculating bubble which corresponds to the pressure distribution on the object, thus resulting on drag coefficient of the object. It is also suggested that future research on recirculating bubble area to be done in order to assure other recirculating bubble properties on drag reduction.

\section{References}

1. B. R. Munson, D. F. Young, and T. H. Okiishi, Fundamentals of Fluid Mechanics. John Wiley, (1998).

2. V. Uruba, P. Jonáš, and O. Mazur, "Control of a channel-flow behind a backward-facing step by suction/blowing," Int. J. Heat Fluid Flow, vol. 28, no. 4, pp. 665-672, (2007).
3. S. Chaligné, T. Castelain, M. Michard, and D. Juvé, "Active control of the flow behind a two-dimensional bluff body in ground proximity," Comptes Rendus Mécanique, vol. 341, no. 3, pp. 289-297, (2013).

4. M. Sano, I. Suzuki, and K. Sakuraba, "Control of turbulent channel flow over a backward-facing step by suction," J. fluid Sci. Technol., vol. 4, no. 1, pp. 188-199, (2009).

5. J. Rajasekaran, "On the flow characteristics behind a backward-facing step and the design of a new axisymmetric model for their study." University of Toronto, (2011).

6. A. S. Monin, "Coherent structure definition," in Akademiia Nauk SSSR Doklady, vol. 318, pp. 853856, (1991).

7. S. C. C. Bailey, R. J. Martinuzzi, and G. A. Kopp, "The effects of wall proximity on vortex shedding from a square cylinder: three-dimensional effects," Phys. Fluids, vol. 14, no. 12, pp. 4160-4177, (2002).

8. K.-B. Chun and H. J. Sung, "Control of turbulent separated flow over a backward-facing step by local forcing," Exp. Fluids, vol. 21, no. 6, pp. 417-426, (1996).

9. T. C. Corke, C. L. Enloe, and S. P. Wilkinson, "Dielectric barrier discharge plasma actuators for flow control*," Annu. Rev. Fluid Mech., vol. 42, pp. 505-529, (2010).

10. G. Nati, M. Kotsonis, S. Ghaemi, and F. Scarano, "Control of vortex shedding from a blunt trailing edge using plasma actuators," Exp. Therm. Fluid Sci., vol. 46, pp. 199-210, (2013).

11. S. G. Pouryoussefi, M. Mirzaei, and M. Hajipour, "Experimental study of separation bubble control behind a backward-facing step using plasma actuators," Acta Mech., vol. 226, no. 4, pp. 11531165, (2015).

12. G. Schewe, "Reynolds Number Effects in Flow Around a Rectangular Cylinder With Aspect Ratio 1: 5," in Proceedings of the Fifth European and African Conference on Wind Engineering, Florence, Italy, (2009).

13. Harinaldi, Budiarso, and J. Julian, "The effect of plasma actuator on the depreciation of the aerodynamic drag on box model," in AIP Conference Proceedings, vol. 1737, no. 1, p. 40004, (2016). 